\title{
Acoustic graphene plasmons advantage: in-plane strong scattering of infrared light by steps at the atomic level
}

\section{Ni Zhang}

Nankai University

\section{Weiwei Luo}

Nankai University

Lei Wang

Nankai University

Jiang Fan

Nankai University

Wei Wu

Nankai University

Mengxin Ren

Nankai University

\section{Xinzheng Zhang}

Nankai University https://orcid.org/0000-0001-8128-3273

Wei Cai ( $\square$ weicai@nankai.edu.cn )

Nankai University

Jingjun Xu

Nankai University

\section{Article}

Keywords: acoustic graphene plasmons, scattering-type scanning near-field optical microscopy, infrared light

Posted Date: January 28th, 2021

DOl: https://doi.org/10.21203/rs.3.rs-150387/v1

License: (c) (1) This work is licensed under a Creative Commons Attribution 4.0 International License.

Read Full License 
Version of Record: A version of this preprint was published at Nature Communications on February 21st, 2022. See the published version at https://doi.org/10.1038/s41467-022-28614-z. 


\title{
Acoustic graphene plasmons advantage: in-plane strong scattering of infrared light by steps at the atomic level
}

\author{
Ni Zhang ${ }^{1}$, Weiwei Luo ${ }^{1}$, Lei Wang ${ }^{2}$, Jiang Fan $^{1}$,Wei Wu ${ }^{1}$, Mengxin \\ Ren $^{1}$, Xinzheng Zhang ${ }^{1}$, Wei Cai ${ }^{1,3}$, Jingjun $\mathrm{Xu}^{1}$ \\ ${ }^{1}$ The Key Laboratory of Weak-Light Nonlinear Photonics, Ministry of Education, School of \\ Physics and TEDA Institute of Applied Physics, Nankai University, Tianjin 300457, China \\ ${ }^{2}$ College of Physics and Electronic Engineering, Xinyang Normal University, Xinyang 464000, \\ China \\ ${ }^{3}$ Collaborative Innovation Center of Extreme Optics, Shanxi University, Taiyuan, Shanxi \\ 030006, People's Republic of China \\ E-mail: weicai@nankai.edu.cn, and jjxu@nankai.edu.cn
}

\begin{abstract}
.
Acoustic graphene plasmons (AGPs) have an extreme level of field confinement and low loss in the mid-infrared and terahertz spectra, which have been applied for quantum effect exploration and ångström-thick material sensing. However, up to now, it is still a lack of exploration of the in-plane scattering of AGPs, though it is essential for the manipulation and utilization of ultraconfined optical field down to atomic level. In this work, by using scattering-type scanning near-field optical microscopy (s-SNOM), the mid-infrared AGPs, which are strongly scattered by atomic level height steps, were imaged in real-space. Particularly, even though the step height of the scatterer is four orders of magnitude lower than the incident free wavelength, strong scattering of AGPs still was achieved and can be attributed to larger reflectivity of AGPs than that of the traditional graphene plasmons (GPs). In addition, the scattering of AGPs by individual scatterers can be controlled via electrical back gating, in which a high fringe contrast up to about $82 \%$ was achieved. Our work suggests a feasible way to control extremely confined optical fields with atomic level height nanostructures, which can be used for ultra-compacted strong light-matter interactions, e.g. photodetector, biosensing, and strong coupling effects.
\end{abstract}

\section{Introduction}

Graphene plasmons (GPs)-electromagnetic fields coupled to charge carrier oscillations, have attracted a great deal of attention owing to their short wavelengths, strong field confinement, and electrical tunability [1-5]. These outstanding properties make GPs great potential applications for controlling electromagnetic waves on the nanometer scale [6], such as highly integrated sensitive spectroscopy [7], modulators [8], and detectors [9-11]. Moreover, by placing the graphene sheet close to a metallic surface, the GPs hybridize with their mirror image, leading to an electromagnetic mode comprising anti-phase charge oscillations in the graphene and the metal [12]. In contrast to GPs, whose energy scales with the square root of their momentum, this new hybridized mode satisfies linear energy versus momentum dispersion, which is named 
acoustic graphene plasmons (AGPs) [13-15]. AGPs lead to extremely strong confined and enhanced electric fields inside the gap between the two materials, which can be confined in-plane extensively to almost $1 / 300$ of their equivalent free-space wavelength [16]. Besides, AGPs are less damped than traditional GPs [17]. In further, by taking advantage of the plasmon confinement down to the length scale of atomic level, quantum effects have been explored with AGPs $[18,19]$. Besides, the extreme field concentration provided by AGPs leads to improved sensitivity of infrared molecular vibrational spectroscopy down to ångström-thick material layers [12,20]. In addition, AGPs can boost nonlinear processes, such as second-harmonic generation or four-wave mixing [21], providing a platform to study enhanced nonlinear light-matter interactions.

Yet, so far the experimental investigation of AGPs has been limited to the realization and detection of ultrastrong optical confinement, and further validated its fundamental applications. The exploration of the scattering of AGPs is still missing, though this is a fundamental prerequisite for in-plane control of the ultraconfined fields of AGPs. Here, relying on scatteringtype scanning near-field optical microscopy (s-SNOM), mid-infrared AGPs modes in h-BN encapsulated graphene deposited on top of Au surface with atomic level thickness steps were imaged and analyzed. Because of the extremely confined electromagnetic fields of AGPs, strong plasmon scattering is observed despite the step height is as low as $3 \mathrm{~nm}$, which is about four orders of magnitude lower than the incident free space wavelength. Moreover, the distribution of near-fields for AGPs can be efficiently tuned in situ by gating the graphene. Our work provides an efficient method to control plasmons by nanostructures with atomic level height. As a result, by engineering substrates vertically in atomic scale, like nano-etching or stacking layered materials, a variety of unique physical phenomena such as photonic crystal [22,23], Anderson localization [24,25], and functionalized optical devices [26,27] based on AGPs can be realized, finding potential applications including spectroscopy, sensing, and optoelectronics.

\section{Experiment and Results}

The schematic of our mid-infrared nanoimaging experiments on AGPs is shown in Fig. 1(a). The heterostructure devices consist of a monolayer graphene flake encapsulated between two layers of hexagonal boron nitride (h-BN). The h-BN $(2 \mathrm{~nm}) /$ graphene/h-BN $(6 \mathrm{~nm})$ heterostructure was assembled by the polymer-free van der Waals assembly technique [28]. Near-field imaging was realized by s-SNOM which is based on a metallic atomic force microscopy (AFM) tip [29-31]. The infrared laser with a wavelength of $10.653 \mu \mathrm{m}$ is focused on the tip, and the sharp tip apex provides wave vector matching between plasmons and incident photons, wherein the incident light is partly converted to plasmons $[32,33]$. The interference between the incident light and plasmon waves reflected back from graphene edges results in fringes with a period of one-half of the polariton wavelength.

Here, we placed the heterostructure on top of a $50 \mathrm{~nm}$ thick Au gate, where the bottom layer of h-BN acts as a spacer and the plasmons in graphene are supposed to hybridize with their mirror image to form AGPs. The propagating AGPs in our devices are visualized by infrared nano-imaging at room temperature, as illustrated in Fig. 1(b) and Fig. 1(e) at the back gate voltages -2 and $-4 \mathrm{~V}$, respectively. The most remarkable feature of the images is that the entire field of view (over $1.5 \mu \mathrm{m} \times 1 \mu \mathrm{m}$ ) is filled with plasmon interference fringes, which have never been observed at room temperature except for graphene under liquid-nitrogen temperatures [34]. Moreover, these interference fringes are disorderly distributed and vary significantly at different gate voltages. It is well known that the interference between the in-plane scattering of propagating plasmons will result in fringes. To determine the scattering sources, the topography of the gold substrate is analyzed. AFM image of the uncovered gold surface reveals randomly distributed holes with depths around $6 \mathrm{~nm}$ (see Fig. S3(a) in supplementary). Correspondingly, the morphology on the encapsulated sample region shows similar shaped but shallower holes, indicating a suspended device on the uneven substrate illustrated in Fig. 2(a). 
Actually, suspended graphene placed on a porous substrate has been studied intensively for potential micro elctro-mechanical applications $[35,36]$. This kind of holes can be the scattering sources for the observed plasmon fringes, where a similar experiment but with a much larger depth of $180 \mathrm{~nm}$ has been reported [37]. Furthermore, to distinguish which these holes presented in the substrate here might stem from, i.e., non-uniform gold evaporation or possible chemical pollution during lift-off process, analysis techniques including scanning Electron Microscope (SEM) and Energy-dispersive X-ray Spectroscopy (EDS) were used to characterize the atomic composition of the sample (see Fig. S4 in the supplementary). By comparing the characteristic peak intensities and atomic percentages of different elements, it can be deduced that the plasmon scattering is caused by the holes formed by gold. In other words, the metallic holes formed during nonuniform evaporation play the role of plasmon scattering sources. Because the wavelength of the plasmon depends on the gate voltages, the variation of interference fringes between Fig. 1(b) and Fig. 1(e) can be understood as that different plasmon interaction appears between scatterers with fixed spatial distribution.

To further confirm that the fringes originate from the interference of AGPs, the results of the Fourier transformed near-fields are shown in Fig. 1(c) and Fig. 1(f). The well fitted concentric circle in momentum space indicates that the chaotic fringes have wave vectors of identical magnitude but widely distributed directions. From the radius of the circles, the obtained fringe periods $\Lambda$ in Fig. 1(b) and Fig. 1(e) are 56 and $67 \mathrm{~nm}$ through $\Lambda=2 \pi /|k|$, respectively. Simultaneously, the plasmons at the boundary of the h-BN encapsulated graphene with voltages of -2 and $-4 \mathrm{~V}$ were also measured and the half wavelengths are approximately 54 and $68 \mathrm{~nm}$, which are consistent with the above obtained periods. Besides, the electrodynamic response of $\mathrm{h}-\mathrm{BN} /$ graphene/h-BN with different gate voltages is vividly illustrated by its dispersion relation [Fig. 1(d)], where the plasmon wavelength $\lambda_{p} \propto E_{F} \propto \sqrt{|n|} \propto \sqrt{\mathrm{U}}$, where $\mathrm{U}$ is back gate voltage between $\mathrm{Au}$ and graphene. The white circles on the color map are the experimental results, corresponding to voltages of $-1,-2,-3$, and $-4 \mathrm{~V}$, respectively, showing good agreement with theoretical ones. A small discrepancy may come from that the near-field images are dominated by the first two peaks close to the scattering sources, where the peak-peak distance is a bit larger than the half plasmon wavelength [4]. This confirms further that the AGPs are the source of the interference fringes.

As revealed by the AFM morphological comparison between the sample (left panel of Fig. 2(a)) and the bare Au substrate, we know that the encapsulated region is partially suspended on the holes. Therefore, a simplified theoretical model is adopted to explore the physical reason why strong scattering of AGPs happens by such shallow steps (approximately $3 \mathrm{~nm}$ ) on the $\mathrm{Au}$ substrate. The scattering source of AGPs can be approximately imitated as holes in the metallic surface. In further, instead of considering very complex three-dimension (3D) model, the reflectivity of traditional GPs and AGPs in 2D for a single scattering boundary was calculated through full-wave electromagnetic simulations by utilizing Comsol Multiphysics. Although the simplified model cannot provide quantized scattering efficiency for the scatterer in our sample, it can offer clues for understanding the physics behind it. The schematic diagram of the calculation model is shown in the right panel of Fig. 2(a). In the simulation, the scattering boundary is described by a step. The height of the step is chosen as $3 \mathrm{~nm}$. And $\theta$ represents the steepness of the step, where $\theta=0^{\circ}$ corresponds to the vertical boundary. For both cases, the h-BN (2 $\mathrm{nm}) /$ graphene/h-BN(6 nm) heterostructure is placed directly on top of the step. The GPs and AGPs with the same amplitude are input from the left port. Figure 2(b) shows the magnetic field profile of $|\mathbf{H}|$ during reflection at $\theta=0^{\circ}$. One can clearly see that for the steps with the same depth, AGPs show an advantage over GPs, reflecting significantly while GPs hardly reflect. Moreover, the calculated amplitude reflectivity under different gate voltages is shown in Fig. 2(c) and Fig. 2(d), which corresponds to GPs and AGPs, respectively. It can be known that, for a step with a height of $3 \mathrm{~nm}$, the reflectivity of GPs is less than $3.8 \%$, but it can reach 
(a)

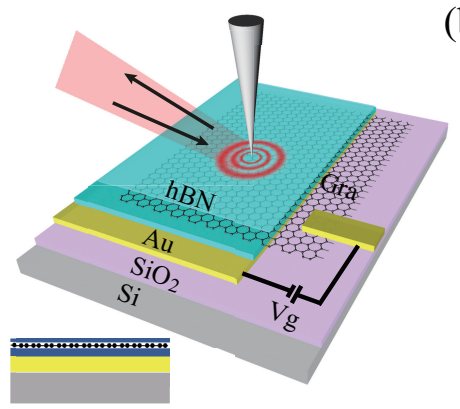

(d)

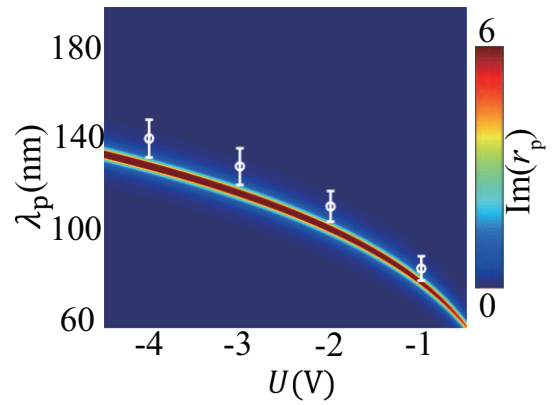

(b)

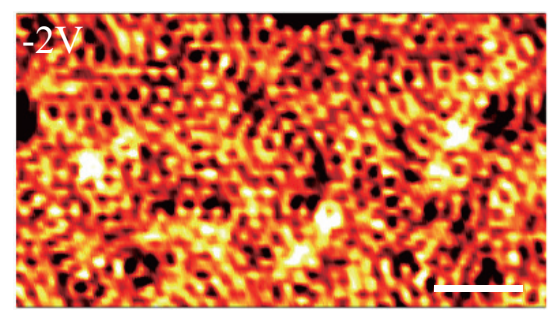

(e)

(c)

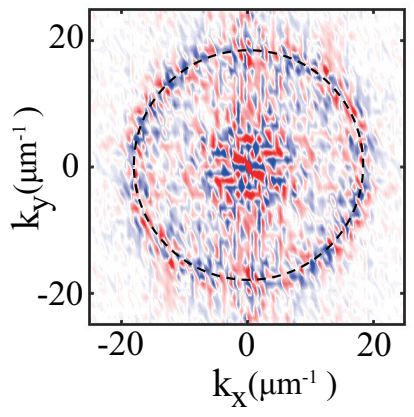

(f)

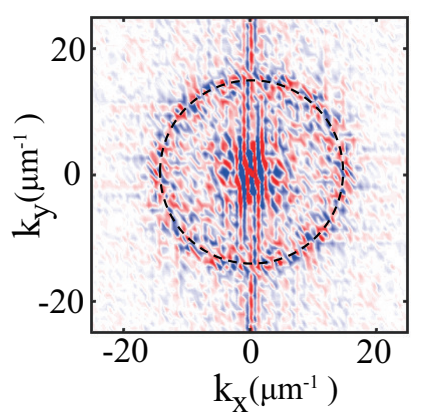

Figure 1. Structure and the near-field response of an AGP device. (a) Schematic of the AGP device, which is composed of h-BN $(2 \mathrm{~nm}) /$ graphene/h-BN $(6 \mathrm{~nm})$ heterostructure placed on $\mathrm{Au}$ $(50 \mathrm{~nm}) / \mathrm{SiO}_{2}(300 \mathrm{~nm}) / \mathrm{Si}$ substrate. The inset shows the cross-section view of the AGPs device. Real space near-field image of the AGPs at $-2 \mathrm{~V}$ (b) and its corresponding wavevectors in Fourier space (c). (d) The wavelength and back gate voltage dependent dispersion of AGPs modes. The white circles represent the experimental results. (e) Near-field image of the AGPs at $-4 \mathrm{~V}$ and the corresponding Fourier transform (f). The wavelength of excitation light is $\lambda=10.653 \mu \mathrm{m}$. Scale bars: $300 \mathrm{~nm}$.

about $13 \%$ for AGPs. This comes from much larger vertical field localization in the gap between metal and graphene for AGPs compared to GPs, providing a great advantage for AGPs. It is worth mentioning that large reflection of traditional GPs can only happen at nanometer scale when the incontinuity of electronic conductivity of graphene is realized [38], however, this is not the requirement for AGPs.

Instead of the collective plasmon interaction induced by randomly distributed scattering sources, the plasmon response of individual scattering source is important as well. To clearly understand the scattering behavior of individual scatterers, another sample showing spatially well-separated scatterers was studied. The sample was assembled in the same way as above, and the thicknesses of the top and bottom h-BN layers are 2 and $5.5 \mathrm{~nm}$, respectively. Figure 3(a) shows the near-field image with sample region over a $2.6 \mu \mathrm{m} \times 2 \mu \mathrm{m}$ area at a gate voltage of 2.9 $\mathrm{V}$. The black dashed line on the right marks the boundary of the h-BN encapsulated graphene. One can find many independent scatterers exist on the near-field image. And one of them is chosen for an in-depth study as enclosed by the black dashed square in Fig. 3(a). The height of the scatterer is approximately $3 \mathrm{~nm}$ with the width of $100 \mathrm{~nm}$ (Fig .S7 in supplementary), which is consistent with previously proposed theoretical model [Fig. 2(a)]. Plasmon near-field mappings on the selected region at gate voltages of 2.2 and $2.9 \mathrm{~V}$ are shown in Fig. 3(b). Compared with the near-field image at $2.2 \mathrm{~V}$, the intensity of the fringes increases, and more fringes become visible at the gate voltage $2.9 \mathrm{~V}$. Furthermore, the spectral profile of different gate voltages across the same scatterer center was extracted along the black dashed line in 
(a)

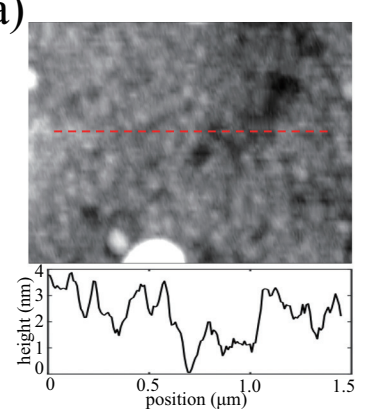

(c)

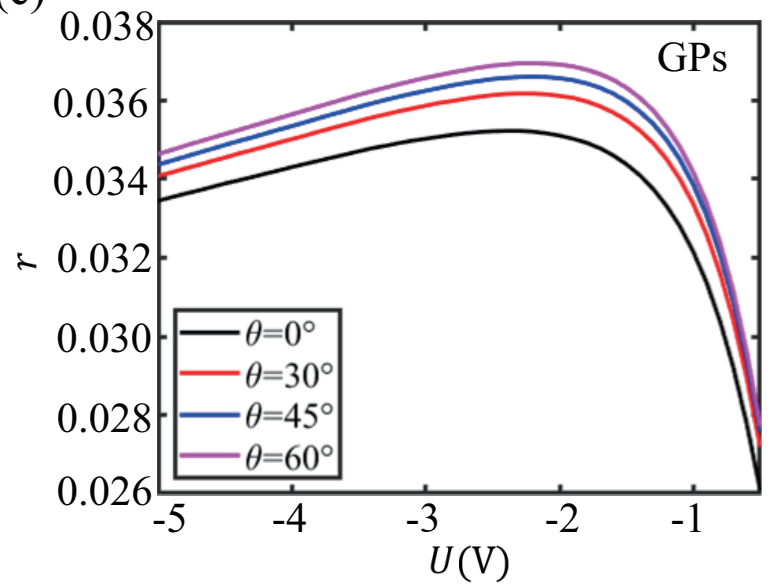

(b)

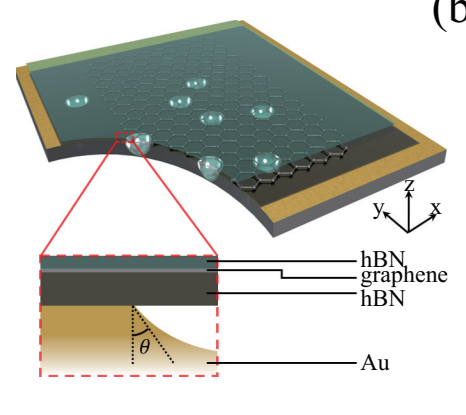

(d)
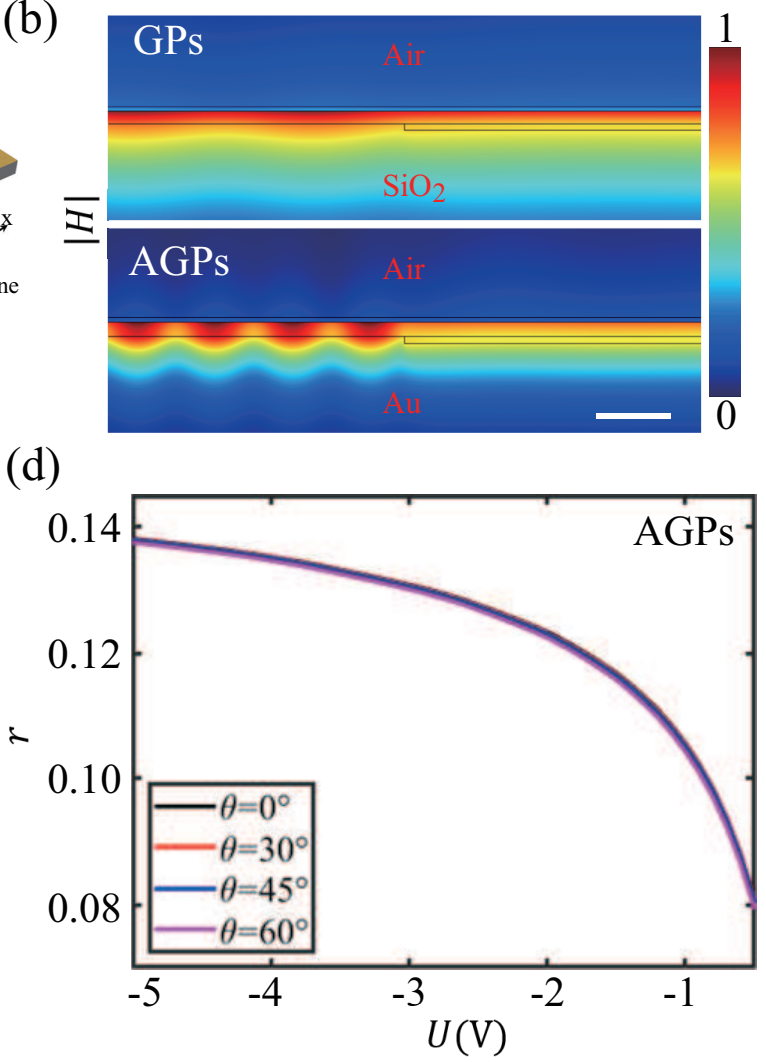

Figure 2. The advantage of in-plane reflectivity of mid-infrared AGPs compared to GPs. (a) Schematic diagram of the simplified calculation model. The left panels show the AFM topography of the h-BN/graphene/h-BN heterostructure placed on the gold surface, where a fluctuation in depth of about $3 \mathrm{~nm}$ can be observed. So the height of the reflection boundaries is set as $3 \mathrm{~nm}$, which is deduced from the topographic differences between the sample and bare gold substrate. (b) The magnetic field profile of $|\mathbf{H}|$ during reflection at $\theta=0^{\circ}$ for GPs and AGPs. Scale bar, $30 \mathrm{~nm}$. (c) and (d) show back gate voltage dependent reflectivity of GPs and AGPs for different steepness of steps, respectively.

Fig. 3(b). Obviously, three distinct peaks are observable when the voltage is $2.9 \mathrm{~V}$, which are hard to be resolved at the voltage of $2.2 \mathrm{~V}$. At the same time, the near-field along the boundary of the encapsulated graphene is shown as solid lines in Fig. 3(d) for comparison. We can find consistent plasmon wavelengths for the substrate scatterers and graphene boundary, and both increase with the gate voltage.

It is noteworthy that obvious interference fringes appear in Fig. 3(a), though the height of these scatterers changes less than $3 \mathrm{~nm}$, which is about four orders of magnitude lower than the incident wavelength of light. Besides, the amplitude of the main fringe of the scatterers is not the same for different gate voltages. Therefore, we further studied the visibility of the main fringe between the scatterers and the boundary of the encapsulated graphene. Three obvious scatterers in the sample are selected and labeled by arrows. Here, the visibility of fringes is defined as $V=\Delta S_{\mathrm{S}} / \Delta S_{\mathrm{R}}$, where $\Delta S_{\mathrm{S}}$ and $\Delta S_{\mathrm{R}}$ are the magnitudes of the first peak in the near-field image relative to the near-field amplitude on graphene far away from it for the scatterer and boundary, respectively. $\Delta S_{\mathrm{R}}$ keeps almost unchanged as the back voltage increases (Fig. S8 in supplementary). However, the visibility gradually increases to 0.82 when the voltage changes 
(a)

(c)
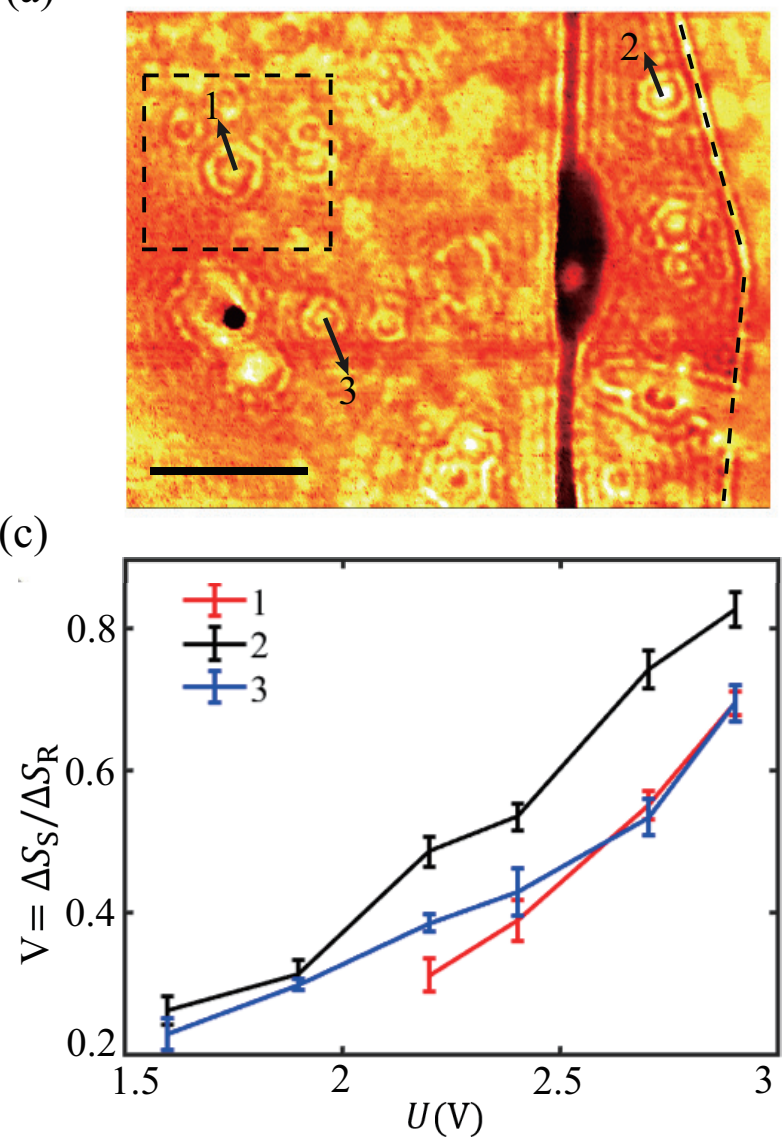

(b)

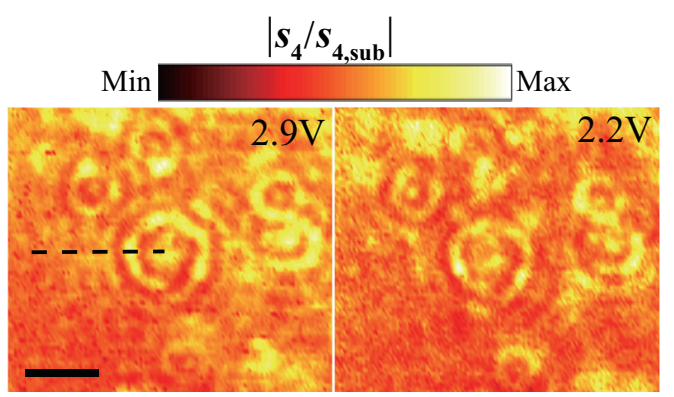

(d)

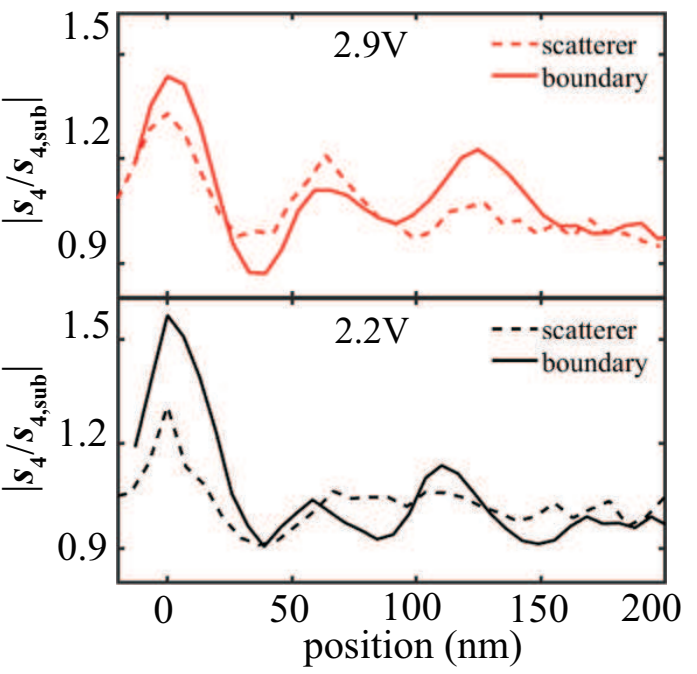

Figure 3. The near-field response of individual scatterers at the wavelength of $\lambda=10.653 \mu \mathrm{m}$. (a) The near-field image of the sample region over a $2.6 \mu \mathrm{m} \times 2 \mu \mathrm{m}$ area under the gate voltage of $2.9 \mathrm{~V}$. The black dashed line and rectangle show the boundary and the selected scatterer area of the h-BN encapsulated single-layer graphene, respectively. Scale bar, $500 \mathrm{~nm}$. (b) Near-field images of the AGPs in the region of the black dashed square in Fig. 3(a) at 2.9 and $2.2 \mathrm{~V}$, respectively. Scale bar, $300 \mathrm{~nm}$. (c) The fringe visibility for the three different scatterers as a function of the gate voltages. (d) Comparison between the normalized near-field signal $S_{4} / S_{4 \text {,sub }}$ of the selected scatterer and the boundary of the graphene as a function of the voltages. The dashed and solid lines represent the scatterer and the boundary, respectively.

from 1.6 to $2.9 \mathrm{~V}$ [Fig. 3(c)]. These features can be understood for the following reasons. Firstly, the scattering of AGPs by holes is more divergent in directions than that for a boundary. As a result, the fringe visibility is always less than 1 . Secondly, the larger visibility with higher voltages can be attributed to larger reflectivity of AGPs, which comes from the increase of Fermi energy of graphene by gate voltages [see Fig. 2(d)]. In further, for different scatterers, the visibility is not exactly the same under the same gate voltage, which may attribute to differences in the size and height of the scatterers.

\section{CONCLUSION}

In conclusion, real-space mid-infrared AGPs were observed in h-BN/graphene/h-BN heterostructure stacked on top of gold film with step height toward to atomic level. Specifically, at room temperature, extensive plasmon interference fringes were surprisingly observed due to 
the scattering from randomly distributed nanometer deep steps of Au film, as not presented in traditional GPs, which can be attributed to that the reflectivity of AGPs is much larger than that of traditional GPs. Moreover, the individual scatterers show the excellent near-field response of AGPs, which has been efficiently tuned by the gate voltage. Based on these properties, AGPs might be exploited in ultra-sensitive optical sensing for nano-scale flatness. Besides, our work opens a venue for efficiently control acoustic plasmons with atomic level height nanostructures, which sheds light onto ultraconfined optical device design by atomic scale substrate engineering, finding potential applications including spectroscopy, sensing, and nanoscale lasers.

\section{Acknowledgment}

This work was supported by Guangdong Major Project of Basic and Applied Basic Research (2020B0301030009), the Program for the National Key R\&D Program of China (2017YFA0305100, 2017YFA0303800), the National Natural Science Foundation of China (91750204, 12074200, 11774185, 12004196, 61775106, 92050114), Changjiang Scholars and Innovative Research Team in University (IRT13_R29), the 111 Project (B07013), the Tianjin Natural Science Foundation (18JCQNJC02100), and Fundamental Research Funds for the Central Universities.

\section{Methods}

I. Samples and devices. Graphene and h-BN were obtained by mechanical cleavage of bulk graphite and hexagonal boron nitride crystal and then transferred to $\mathrm{SiO}_{2}(300 \mathrm{~nm}) / \mathrm{Si}$ substrate. Then by employing a polymer-free van der Waals assembly technique [39], the encapsulated device was assembled. In brief, a thin layer h-BN crystal was used to pick up single-layer graphene and another h-BN crystal in sequence. Then the encapsulated graphene structure was placed on the $\mathrm{Au} / \mathrm{SiO}_{2}(300 \mathrm{~nm}) / \mathrm{Si}$ substrate. Electrical contact to graphene and backgate were realized by pre-defined $\mathrm{Au}$ pads on $\mathrm{Si} / \mathrm{SiO}_{2}$ via UV lithography and electron-beam deposition.

II. Plasmon near-field image. Near-field imaging was achieved by the s-SNOM (Neaspec $\mathrm{GmbH}$ ) based on a metal AFM tip, which is illuminated by infrared light from a $\mathrm{CO}_{2}$ laser. Plasmon reflection at the graphene edges and scatterers produces plasmon interference, which is imaged by recording the light elastically scattered by the tip with a pseudo-heterodyne interferometer. In order to suppress background scattering from the tip shaft and sample, the tip is vibrated vertically with the frequency of around $270 \mathrm{kHz}$, with an oscillation amplitude of about $60 \mathrm{~nm}$, and the fourth-order demodulated harmonics of the near-field amplitude was adopted.

III. Dispersion calculation. The dispersion of AGPs was calculated using a classical electromagnetic transfer matrix method, with a thin-film stack of air/h-BN(2 nm)/graphene/h$\mathrm{BN}(6 \mathrm{~nm}) / \mathrm{Au}(50 \mathrm{~nm}) / \mathrm{SiO}_{2}(300 \mathrm{~nm}) / \mathrm{Si}$. Specifically, Fig.1(d) is obtained by calculating the imaginary part of the reflection coefficient $\left(\operatorname{Im}\left\{r_{p}\right\}\right)$ of the evanescent wave. The conductivity of graphene was given by local random-phase approximation [40] and the h-BN permittivity parameters were shown in Table S1.

\section{Author contributions}

W. C., W. L., and J. X. conceived the idea and supervised the project. N. Z and W. L. performed the experiments. W. W. and M. R. participated in the sample characterization. Theoretical background and simulations were provided by L. W., J. F., and Z. Z.. N. Z., W. L. and L. W. contributed equally to this work. All authors discussed the results and wrote the manuscript.

\section{Competing interests}

The authors declare no competing interests. 


\section{References}

[1] Wunsch B, Stauber T, Sols F and Guinea F 2006 New Journal of Physics 8318

[2] Jablan M, Buljan H and Soljačić M 2009 Physical review B 80245435

[3] Koppens F H L, Chang D E and García de Abajo F J 2011 Nano Letters 11 3370-3377

[4] Fei Z, Rodin A S, Andreev G O, Bao W, McLeod A S, Wagner M, Zhang L M, Zhao Z, Thiemens M, Dominguez G, Fogler M M, Neto A H C, Lau C N, Keilmann F and Basov D N 2012 Nature 487 82-85

[5] Chen J, Badioli M, Alonso-González P, Thongrattanasiri S, Huth F, Osmond J, Spasenović M, Centeno A, Pesquera A, Godignon P, Zurutuza Elorza A, Camara N, de Abajo F J G, Hillenbrand R and Koppens F H L 2012 Nature 487 77-81

[6] Vakil A and Engheta N 2011 Science 332 1291-1294

[7] Rodrigo D, Limaj O, Janner D, Etezadi D, García de Abajo F J, Pruneri V and Altug H 2015 Science 349 $165-168$

[8] Woessner A, Gao Y, Torre I, Lundeberg M B, Tan C, Watanabe K, Taniguchi T, Hillenbrand R, Hone J and Polini M a 2017 Nature Photonics 11 421-424

[9] Cai X, Sushkov A B, Jadidi M M, Nyakiti L O, Myers-Ward R L, Gaskill D K, Murphy T E, Fuhrer M S and Drew H D 2015 Nano Letters 15 4295-4302

[10] Freitag M, Low T, Zhu W, Yan H, Xia F and Avouris P 2013 Nature Communications 41951

[11] Koppens F H L, Mueller T, Avouris P, Ferrari A C, Vitiello M S and Polini M 2014 Nature Nanotechnology $9780-793$

[12] Lee I H, Yoo D, Avouris P, Low T and Oh S H 2019 Nature Nanotechnology 14 313-319

[13] Gu X, Lin I T and Liu J M 2013 Applied Physics Letters 10397

[14] A A P, B R A and A M P 2011 Solid State Communications 151 1627-1630

[15] Alonso-González P, Nikitin A Y, Gao Y, Woessner A, Lundeberg M B, Principi A, Forcellini N, Yan W, Vélez S, Huber A J, Watanabe K, Taniguchi T, Casanova F, Hueso L E, Polini M, Hone J, Koppens F H L and Hillenbrand R 2017 Nature Nanotechnology 12 31-35

[16] Epstein I, Alcaraz D, Huang Z, Pusapati V V, Hugonin J P, Kumar A, Deputy X, Khodkov T, Rappoport T G and Peres N M R a 2020 Science 368 1219-1223

[17] Menabde S, Lee I H, Lee S, Ha H, Heiden J, Yoo D, Kim T T, Lee Y H, Low T, Oh S H and Jang M S 2020 arXiv:2010.01958

[18] Lundeberg M B, Gao Y, Asgari R, Tan C, Van Duppen B, Autore M, Alonso-González P, Woessner A, Watanabe K, Taniguchi T, Hillenbrand R, Hone J, Polini M and Koppens F H L 2017 Science 357 $187-191$

[19] Alcaraz Iranzo D, Nanot S, Dias E J C, Epstein I, Peng C, Efetov D K, Lundeberg M B, Parret R, Osmond J, Hong J Y, Kong J, Englund D R, Peres N M R and Koppens F H L 2018 Science 360 291-295

[20] Chen S, Autore M, Li J, Li P, Alonso-Gonzalez P, Yang Z, Martin-Moreno L, Hillenbrand R and Nikitin A Y 2017 ACS Photonics 4 3089-3097

[21] Rivera N, Kaminer I, Zhen B, Joannopoulos J D and Soljačić M 2016 Science 353 263-269

[22] Joannopoulos J, Johnson S, Winn J and Meade R 1995 Photonic Crystals: Molding the Flow of Light

[23] Joannopoulos J D, Villeneuve P R and Fan S 1997 Solid State Communications 102 165-173

[24] Lagendijk A, Tiggelen B V and Wiersma D S 2009 Physics Today 62 24-29

[25] Chaves A, Peres N and Pinheiro F A 2015 Physical Review B 92195425

[26] Li Z, Yao K, Xia F, Shen S, Tian J and Liu Y 2015 Scientific Reports 512423

[27] Bao Q, Zhang H, Wang B, Ni Z, Lim C H Y X, Wang Y, Tang D Y and Loh K P 2011 Nature photonics 5 411-415

[28] Wang L, Meric I, Huang P Y, Gao Q, Gao Y, Tran H, Taniguchi T, Watanabe K, Campos L M, Muller D A, Guo J, Kim P, Hone J, Shepard K L and Dean C R 2013 Science 342 614-617

[29] Gerber J A, Berweger S, O'Callahan B T and Raschke M B 2014 Physical review letters 113055502

[30] Jiang X, Cai W, Luo W, Xiang Y, Zhang N, Ren M, Zhang X and Xu J 2018 Nanotechnology 29385205

[31] Luo W, Cai W, Xiang Y, Wu W, Shi B, Jiang X, Zhang N, Ren M, Zhang X and Xu J 2017 Advanced Materials 291701083

[32] Woessner A, Lundeberg M B, Gao Y, Principi A, Alonso-González P, Carrega M, Watanabe K, Taniguchi T, Vignale G, Polini M et al. 2015 Nature materials 14 421-425

[33] Fei Z, Andreev G O, Bao W, Zhang L M, McLeod A S, Wang C, Stewart M K, Zhao Z, Dominguez G, Thiemens M, Fogler M M, Tauber M J, Castro-Neto A H, Lau C N, Keilmann F and Basov D N 2011 Nano Letters 11 4701-4705

[34] Ni G X, McLeod A S, Sun Z, Wang L, Xiong L, Post K W, Sunku S S, Jiang B Y, Hone J, Dean C R, Fogler M M and Basov D N 2018 Nature 557 530-533

[35] Zhang W, Ma R, Chen Q, Xia M, Ng J, Wang A and Xie Y H 2016 Applied Physics Letters 108153103

[36] Nagase M, Hibino H, Kageshima H and Yamaguchi H 2013 Applied Physics Express 6055101 
[37] Zhang N, Jiang X, Fan J, Luo W, Xiang Y, Wu W, Ren M, Zhang X, Cai W and Xu J 2019 Nanotechnology 30505201

[38] Chen J, Nesterov M L, Nikitin A Y, Thongrattanasiri S, Alonso-González P, Slipchenko T M, Speck F, Ostler M, Seyller T, Crassee I, Koppens F H L, Martin-Moreno L, García de Abajo F J, Kuzmenko A B and Hillenbrand R 2013 Nano Letters 13 6210-6215

[39] Zomer P J, Guimares M H D, Brant J C, Tombros N and Van Wees B J 2014 Applied Physics Letters 105

[40] Jablan M, Buljan H and Soljacic M 2009 Physical review B 80245435 


\section{Figures}

(a)

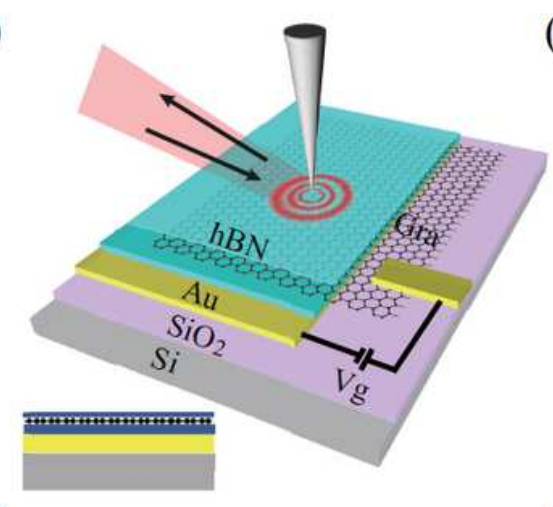

(d)

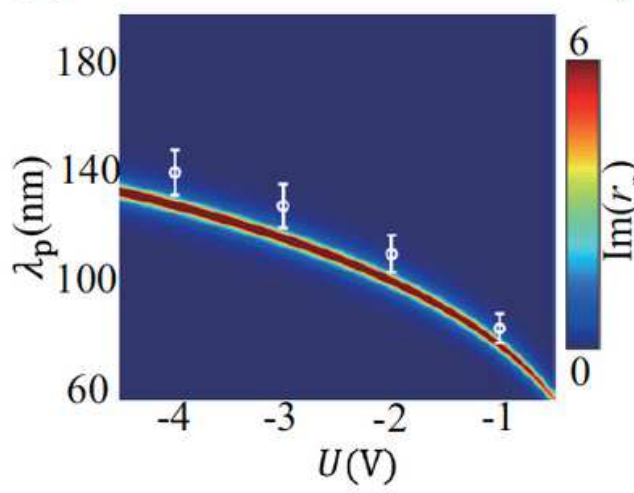

(b)

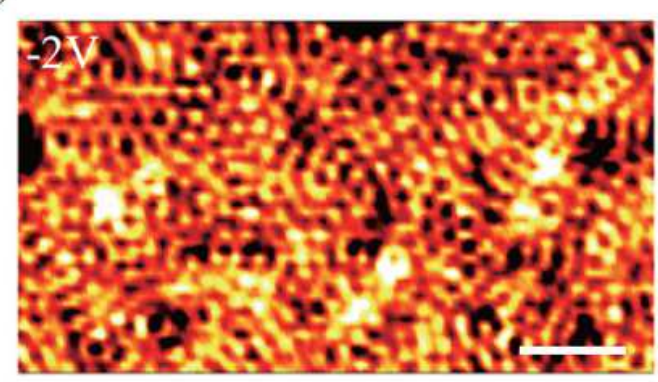

(e)
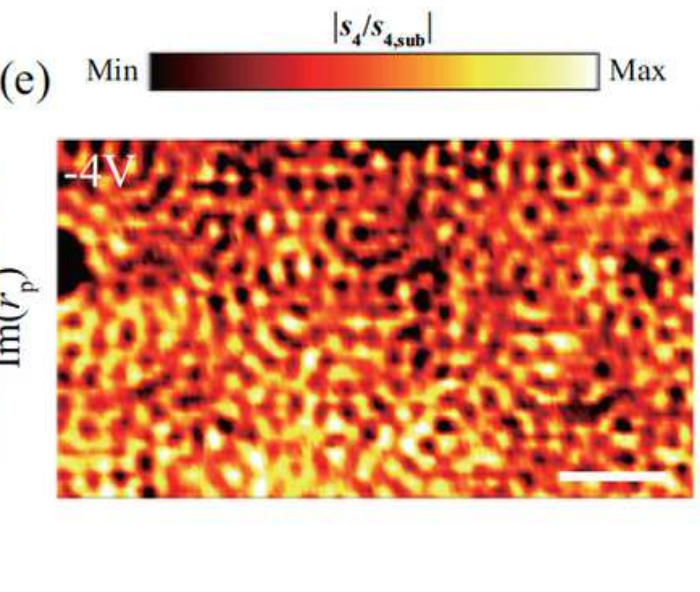

(c)

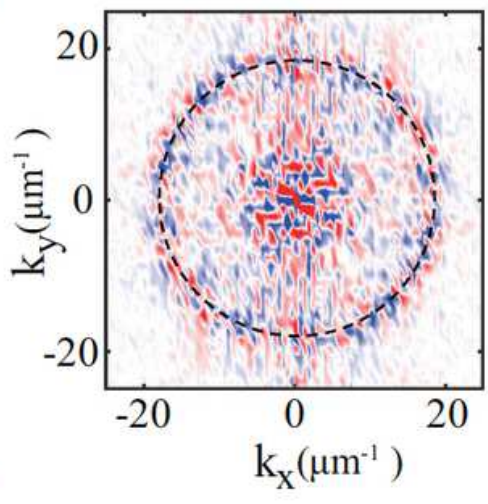

(f)

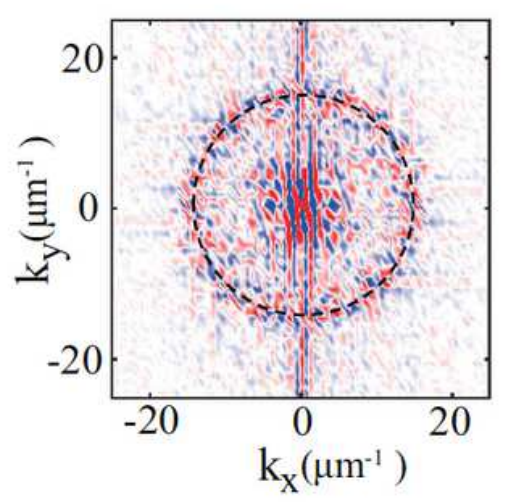

\section{Figure 1}

Structure and the near-field response of an AGP device. (a) Schematic of the AGP device, which is composed of h-BN(2 nm)/graphene/h-BN(6 nm) heterostructure placed on Au $(50 \mathrm{~nm}) / \mathrm{SiO} 2(300 \mathrm{~nm}) / \mathrm{Si}$ substrate. The inset shows the cross-section view of the AGPs device. Real space near-field image of the AGPs at $-2 \mathrm{~V}(\mathrm{~b})$ and its corresponding wavevectors in Fourier space (c). (d) The wavelength and back gate voltage dependent dispersion of AGPs modes. The white circles represent the experimental results. (e) Near-field image of the AGPs at $-4 \mathrm{~V}$ and the corresponding Fourier transform (f). The wavelength of excitation light is $\lambda=10.653 \mu \mathrm{m}$. Scale bars: $300 \mathrm{~nm}$. 
(a)

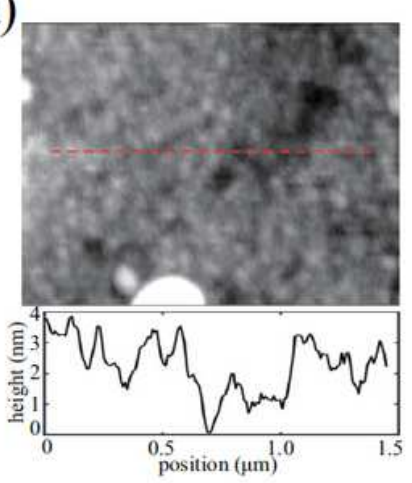

(c)

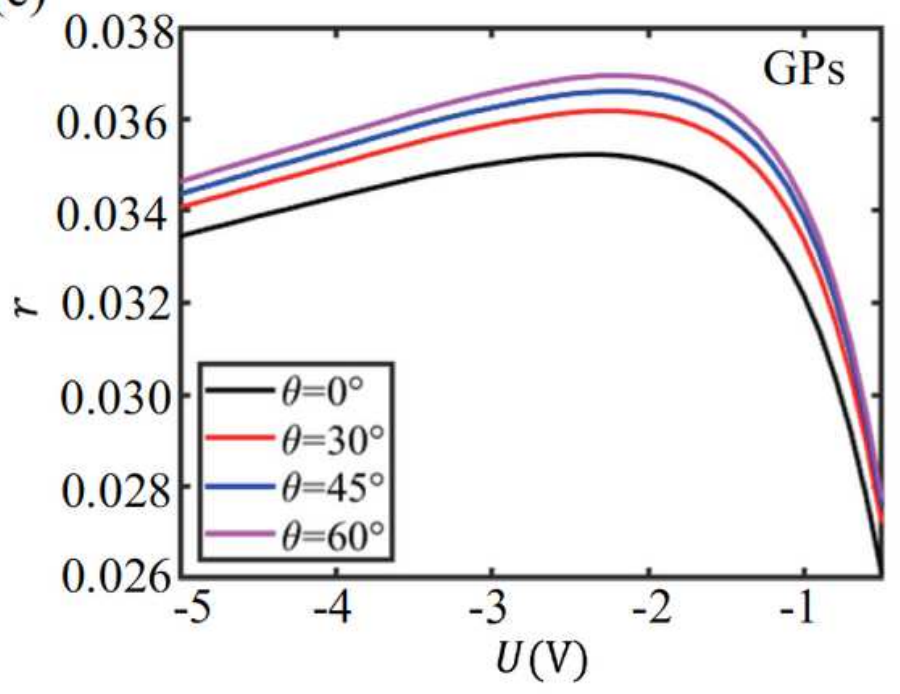

(b)

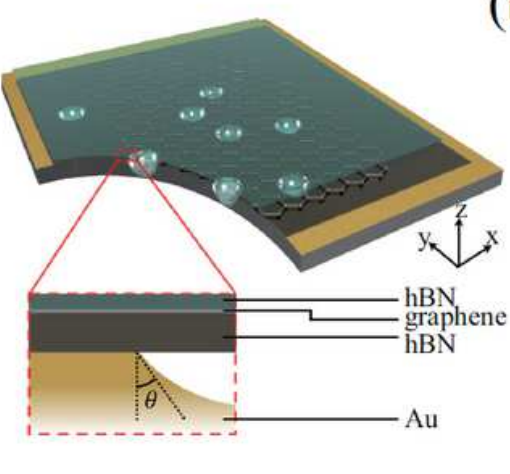

(d)

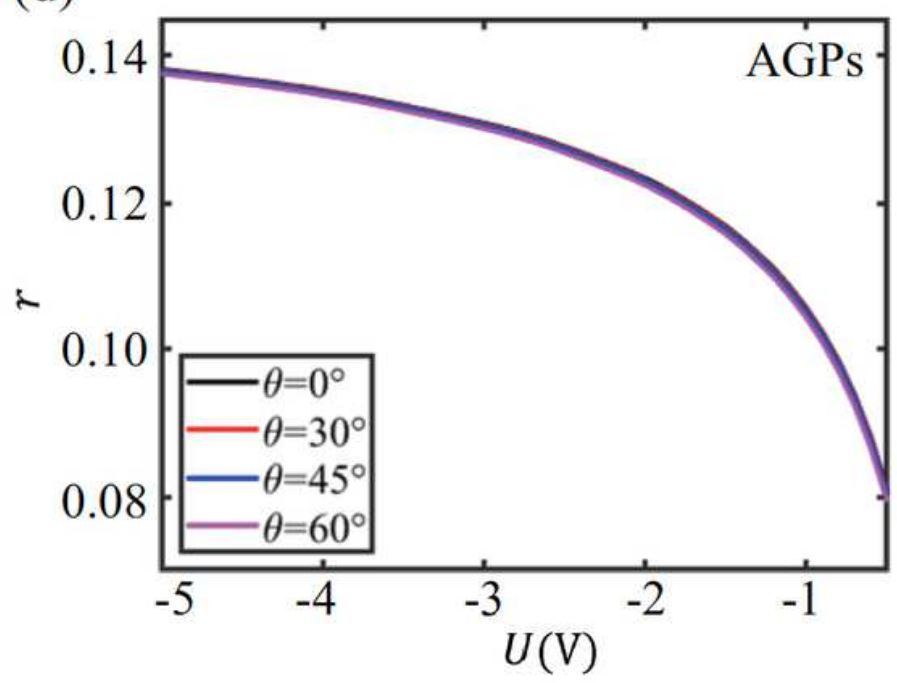

\section{Figure 2}

The advantage of in-plane reflectivity of mid-infrared AGPs compared to GPs. (a) Schematic diagram of the simplified calculation model. The left panels show the AFM topography of the h-BN/graphene/h-BN heterostructure placed on the gold surface, where a fluctuation in depth of about $3 \mathrm{~nm}$ can be observed. So the height of the reflection boundaries is set as $3 \mathrm{~nm}$, which is deduced from the topographic differences between the sample and bare gold substrate. (b) The magnetic field profile of $|\mathrm{H}|$ during reflection at $\theta=0 \otimes$ for GPs and AGPs. Scale bar, $30 \mathrm{~nm}$. (c) and (d) show back gate voltage dependent reflectivity of GPs and AGPs for different steepness of steps, respectively. 
(a)

(c)
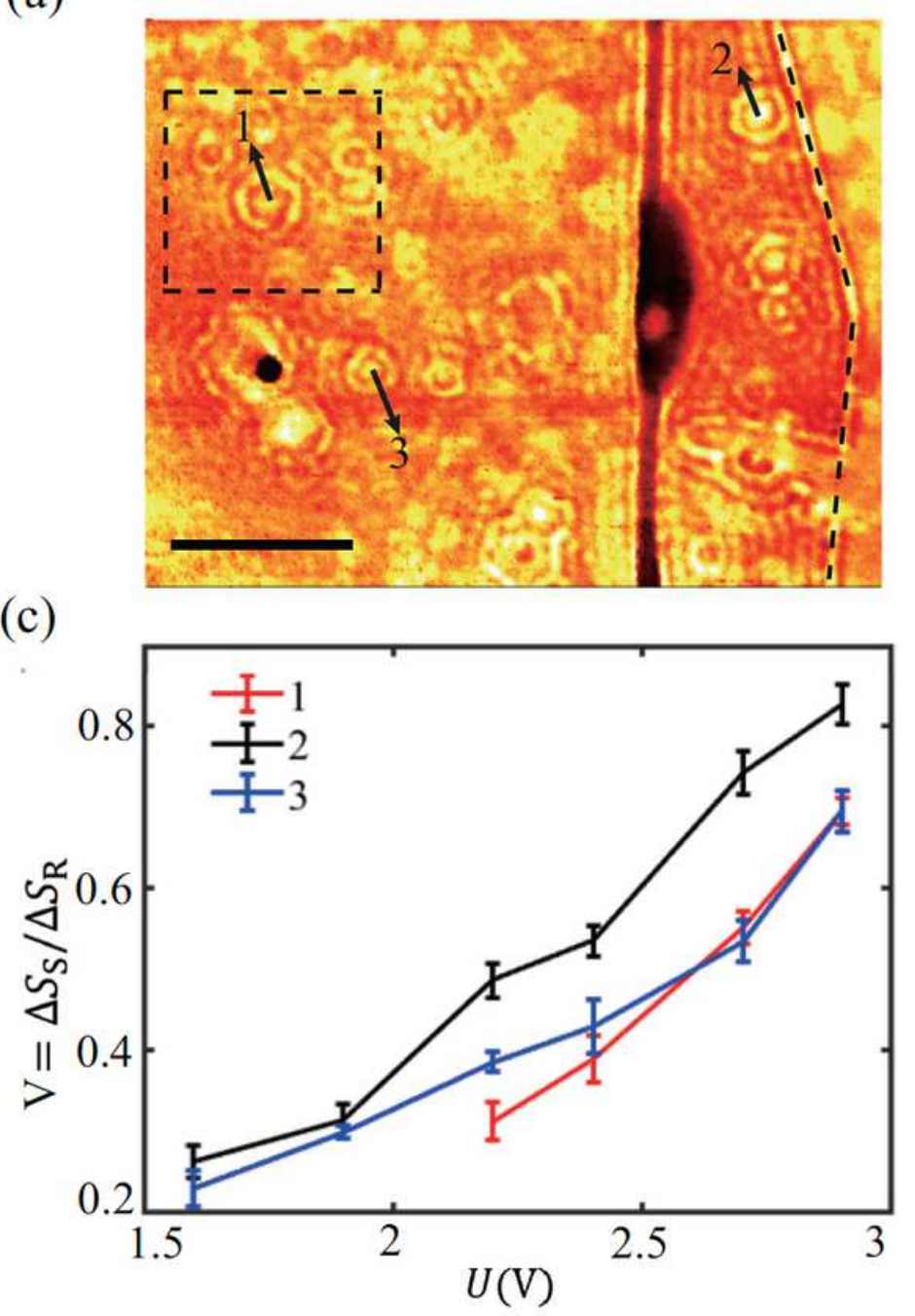

(b)

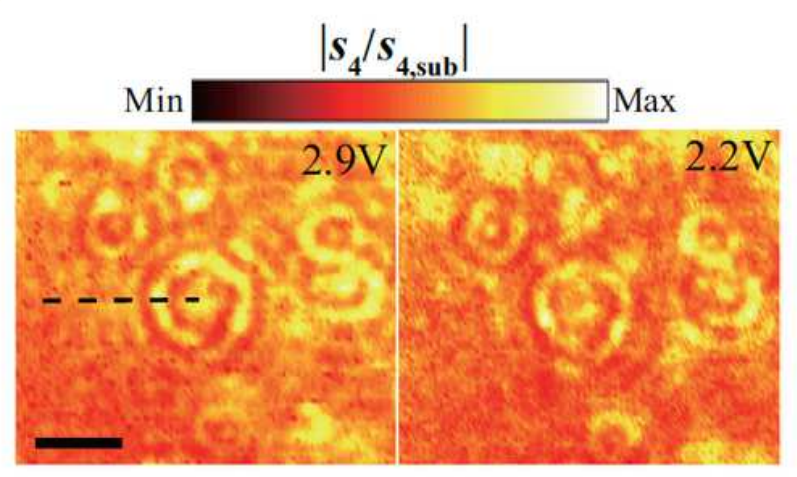

(d)

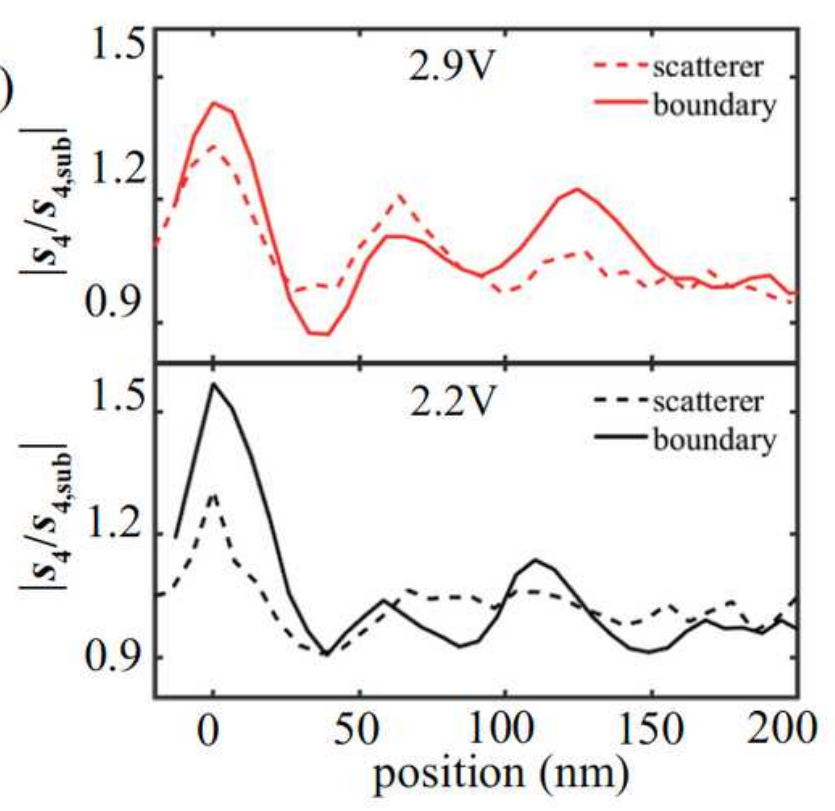

\section{Figure 3}

The near-field response of individual scatterers at the wavelength of $\lambda=10.653 \mu \mathrm{m}$. (a) The near-field image of the sample region over a $2.6 \mu \mathrm{m} \times 2 \mu \mathrm{m}$ area under the gate voltage of $2.9 \mathrm{~V}$. The black dashed line and rectangle show the boundary and the selected scatterer area of the h-BN encapsulated singlelayer graphene, respectively. Scale bar, $500 \mathrm{~nm}$. (b) Near-field images of the AGPs in the region of the black dashed square in Fig. 3(a) at 2.9 and $2.2 \mathrm{~V}$, respectively. Scale bar, $300 \mathrm{~nm}$. (c) The fringe visibility for the three different scatterers as a function of the gate voltages. (d) Comparison between the normalized near-field signal S4/S4,sub of the selected scatterer and the boundary of the graphene as a function of the voltages. The dashed and solid lines represent the scatterer and the boundary, respectively.

\section{Supplementary Files}

This is a list of supplementary files associated with this preprint. Click to download. 
- supportinginformation.pdf 